\title{
INTERNATIONAL UNION OF PURE AND APPLIED CHEMISTRY
}

\author{
ANALYTICAL CHEMISTRY DIVISION \\ COMMISSION ON ELECTROANALYTICAL CHEMISTRY*
}

\section{RECOMENDATIONS FOR NOMENCLATURE OF ION-SELECTIVE ELECTRODES}

\author{
(IUPAC Recommendations 1994) \\ Prepared for publication by \\ RICHARD P. BUCK and ERNÖ LINDNER ${ }^{\dagger}$ \\ ${ }^{1}$ Department of Chemistry, University of North Carolina, Chapel Hill, NC 27599-3290, USA
}

\begin{abstract}
*Membership of the Commission for 1987-1991, when the report was prepared was as follows:
Chairman: M. Senda (Japan; 1987-1989); R. A. Durst (USA; 1989-1991); Vice-Chairman: R. Kalvoda (Czechoslovakia: 1987-1989); M. Senda (Japan; 1989-1991); Secretary: R. A. Durst (USA; 1987-1989); K. M. Kadish (USA; 1987-1991); Titular Members: R. P. Buck (USA; 1989-1993); J. Buffle (Switzerland; 1987-1989); M. Gross (France; 1987-1991); K. Stulik (Czechoslovakia; 1989-1993); K. Tóth (Hungary; 1987-1991); Associate Members: A. M. Bond (Australia; 1989-1993); K. Cammann (Germany; 1989-1993); F. Camoes (Portugal; 1987-1991); N. Davison (UK; 1987-1989); A. Fogg (UK; 1987-1991); H. Kao (China; 1987-1989); R. C. Kapoor (India; 1987-1989); W. Kutner (Poland; 1989-1993); T. Kuwana (U.S.A.; 1989-1991); H. P. van Leeuwen (Netherlands; 1987-1993); M. L'Her (France; 1989-1993); J. G. Osteryoung (USA; 1987-1989); G. Prabhakara Rao (India; 1989-1993); S. Rondinini Cavallari (Italy; 1987-1991); Y. Umezawa (Japan; 1987-1991); E. Wang (China; 1987-1991); National Representatives: G. E. Batley (Australia; 1987-1989); B. Gilbert (Belgium; 1987-1989); H. Y. Chen (China; 1990-1991); A. A. Vlcek (Czechoslovakia; 1987-1989); H. B. Nielsen (Denmark; 1987-1989); H. B. Kristensen (Denmark; 1988-1991); E. Lindner (Hungary; 1987-1991); W. F. Smyth (Ireland; 1987-1989); E. Grushka (Israel; 1987-1989); T. Mussini (Italy; 1989-1991); K. Izutsu (Japan; 1987-1991); A. J. McQuillan (New Zealand; 1987-1991); Z. Galus (Poland; 1987-1991); J. Galvez (Spain; 1987-1991); G. Johansson (Sweden; 1987-1991); G. Somer (Turkey; 1987-1991); A. K. Covington (UK; 1987-1991); J. F. Coetzee (USA; 1987- 1989); W. F. Koch (USA; 1989-1991); I. Piljac (Yugoslavia; 1987-1991).
\end{abstract}

†Visiting Scholar, 1991-1993.

Names of countries given after Members' names are in accordance with the IUPAC Handbook 1991-93; changes will be effected in the 1994-95 edition.

Republication of this report is permitted without the need for formal IUPAC permission on condition that an acknowledgement, with full reference together with IUPAC copyright symbol (C) 1994 IUPAC), is printed. Publication of a translation into another language is subject to the additional condition of prior approval from the relevant IUPAC National Adhering Organization. 


\title{
Recommendations for nomenclature of ion-selective electrodes (IUPAC Recommendations 1994)
}

\begin{abstract}
The rapid growth of interest in the field of ion-selective electrodes (ISEs), within the larger field of electrochemical sensors based on potentiometric, amperometric, and conductometric principles, makes it highly desirable to achieve improved standardization of nomenclature in this area. The previous document, IUPAC Recommendations 1975 (1) has been corrected using recent experimental and theoretical findings. This report does not define or recommend activity standards or calibration procedures using activity standards.
\end{abstract}

\section{GENERAL RECOMMENDATIONS}

\section{Definitions of Terms}

1. Activity, Activity Coefficient and Concentration. Refer to Reterence (2).

2. Calibration Plot. This is a plot of the cell emf (electromotive force measured as ion-selective electrode potential minus external reference electrode potential) of a given ion-selective electrode cell assembly (ion-selective electrode combined with an identified external reference electrode) $v s$. the logarithm of the single ionic activity (concentration) of a given species $(3,4,5)$. For uniformity, it is recommended that the cell emf be plotted on the ordinate (vertical axis) with the more positive emfs at the top of the graph and that $p a_{A}$ (-log activity of the species measured, $\mathrm{A}$ ) or $\mathrm{p} c_{\mathrm{A}}(-\log$ concentration of the species measured, A) be plotted on the abscissa (horizontal axis) with increasing activity to the right. Comment: The cell emf is composed of identifiable interfacial and bulk potential differences arising from the membrane, reference electrodes, and junctions if present (see below). When referring to the potential difference at an ISE membrane interface, or across the ISE membrane including bulk and two interfaces, the terms interfacial potential difference, and ISE or membrane potential difference should be used to distinguish a portion of the cell emf under study.

3. Combination Electrode. An ion-selective electrode and an external reference electrode combined in a single unit, thereby avoiding the need for a separate external reference electrode. (See also terms $\# 17 \mathrm{a}$ and $17 \mathrm{~b}$ Reference electrode).

4. Detection Limit. A calibration plot ordinarily has the shape shown in Fig. 1. By analogy with definitions adopted in other fields, a limit of detection could be defined as the concentration for which, under the specified conditions, the cell emf, $E$, deviates from the average emf in region I by a multiple of the standard error of a single measurement of the emf in this region I. The multiple selected depends on a statistical significance level selected.

The fundamental difference in the nature of ISE potentiometry (their logarithmic response) as compared to linear methods, justifies another type of definition of detection limit. For the sake of practical convenience, a simpler (and more convenient) definition is recommended at this time. The practical lower limit of detection may be taken as the activity (or concentration) of substance A at the point of intersection of the extrapolated linear midrange and final low concentration level segments of the calibration plot, as shown in Fig. 2.

For electrodes that become insensitive to high ionic activities, or the slope of the curve changes its sign, an upper limit of determination may be taken as the activity (or concentration) of $\mathrm{A}$ at the point of intersection of the extrapolated midrange linear segment and the limiting high activity response. Comment: The reliability of the detection limit data, determined by the cross point method, is illustrated in Fig. 2. The reliability depends on:

1. The standard deviation of a single potential measurement in both linear regions (it need not be the same in the Nernstian and concentration-independent regions);

2. The number of data points taken into account to determine the parameters of the linear sections;

3. The concentration range used to determine the parameters of the linear sections.

Based on the above, beside the experimental conditions (i.e., composition of the solution, the 


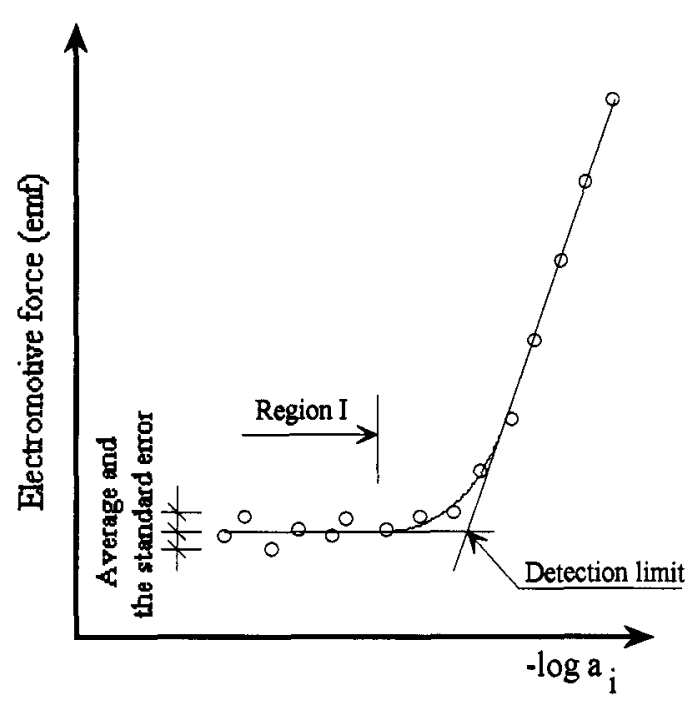

Fig. 1. Calibration plot of an ion-selective electrode.

( $a_{\mathrm{i}}$ denotes the single ion activity of the primary ion, i.)

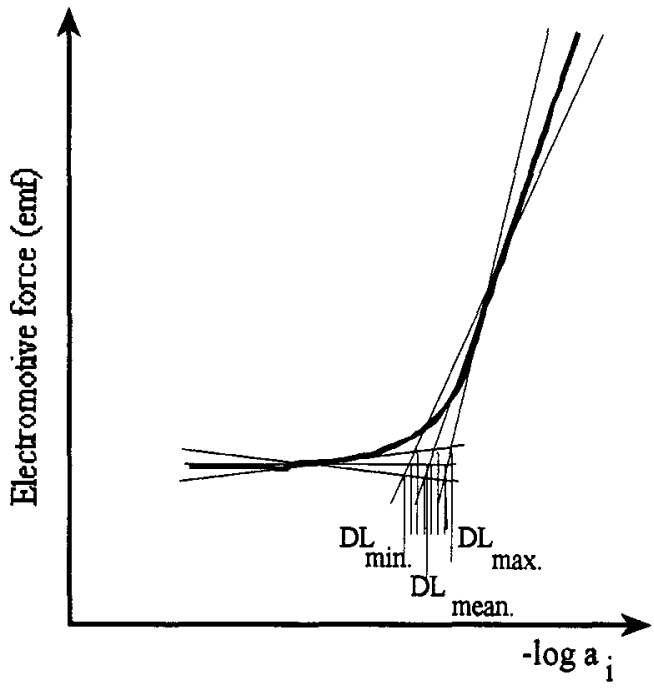

Fig. 2. Determination of the detection limit (DL) of ion-selective electrodes.

( $D L_{\text {mean }}$ is the most probable value of the $\mathrm{DL}$ determined from the cross point of the lines fitted to the linear segments of the emf vs. $\log a_{i}$ curve where $a_{1}$ denotes the single ion activity of the primary ion, i. $\mathrm{DL}_{\min }$. and $\mathrm{DL}_{\max }$ are the lowest and highest concentration values determined as DL taking into account the minimum and maximum slope values ( $95 \%$ confidence values) of the lines fitted to the experimental data.)

Fig.3. Definition and determination of response time $t(\Delta E / \Delta t)$, the time which elapses between the instant when an ion-selective electrode and a reference electrode (ISE cell) are brought into contact with a sample solution (or at which the activity of the ion of interest in a solution is changed) and the first instant at which the emf/time slope $(\Delta E / \Delta t)$ becomes equal to a limiting value selected on the basis of the experimental conditions and/or requirements concerning the accuracy (e.g. $0.6 \mathrm{mV} / \mathrm{min}$ ). 
history and preconditioning of the electrode, stirring rate, etc.) used for the determination of the detection limit, the details of the measured data, (e.g. number of measurements, standard deviation of a single measurement, etc.) and of the evaluation method, should be given as well. However, the description of the experimental conditions and the evaluation method offers only a possibility to reproduce the data, but does not help in the comparison of data collected under different conditions. Accordingly, the experimental conditions and the evaluation method should be standardized if possible. For the determination of the lower detection limit, for example:

1. The potential data used for the determination of detection limit should be measured a minimum of three times: from low to high, high to low and low to high concentrations again.

2. Data used to determine the linear sections of the plot should be collected from a concentration range two orders of magnitude higher, and two orders of magnitude lower, including the concentration of the cross point. A minimum of five data points in both regions should be taken into account within this concentration range to determine the parameters of the linear sections.

3. The parameters of the determined linear sections should be given with their standard deviations.

4. Based on the above, beside the most probable values of the detection limits $\left(\mathrm{DL}_{\text {mean }}\right)$, the confidence interval should be given as well ( $\mathrm{DL}_{\min }, \mathrm{DL}_{\max }$.).

5. If the determination of data points below the detection limit is problematic, (too long response time, too bad reproducibility, etc.) a line parallel to the $x$-axis should be drawn through the mean potential value measured in the solution of lowest concentration, instead of fitting a line through some data in the curved region of the calibration plot. The detection limit should be determined similarly as above, only the data $\mathrm{DL}_{\max }$. should be given as detection limit as $\mathrm{DL}<$ numerical value \pm standard deviation.

5. Drift and Standard Deviation. Drift is the slow non-random change with time in the emf of an ionselective electrode cell assembly maintained in a solution of constant composition and temperature. The determination of the drift is carried out by a linear curve fitting on the data set collected in a given period of time in a solution of constant composition and temperature. The slope of the emf $v s$. time line is called drift. The random emf deviations around the line define the standard deviation of the measured potential data.

6. Hysteresis and Reproducibility. Hysteresis or electrode memory occurs when there is a difference between the emf first observed in a solution containing a concentration of $\mathrm{A}$ and a second observation of the emf in the same solution after exposing the electrode to a different concentration of $A$. The systematic error is generally in the direction of the concentration of the solution in which the electrode was previously immersed. Hysteresis is thought to be a kinetic process. Normal, reversible responses are expected when sufficient time is allowed for the system to return to its initial condition.

The standard deviation of emf data collected in a series of emf measurements in solutions of different concentrations of $A$ (after removal and washing and/or wiping of the electrodes) is called reproducibility. If the electrode does have a distinct hysteresis, the reproducibility will be poor. Successive emf measurements in solution A, provides a measure of drift and standard deviation of the electrode, not a measure of hysteresis.

7. Interfering Substance. This may be any substance, other than the ion being measured, whose presence in the sample solution affects the measured emf of a cell. Interfering substances fall into two classes: "electrode/electrochemical" interferences and "chemical" interferences. Examples of the first class include:

a. Those substances which give a similar response to the ion being measured and whose presence generally results in an apparent increase in the activity (or concentration) of the ion to be determined (e.g., $\mathrm{Na}^{+}$for the $\mathrm{Ca}^{2+}$ electrode).

b. Electrolytes present at a high concentration that give rise to appreciable liquid junction potential differences or results in a significant activity coefficient decrease, or incipient Donnan exclusion failure.

The second class of substances that should be recognized as chemical interferences includes:

c. Species that interact with the ion being measured so as to decrease its activity or apparent concentration. The electrode continues to report the true activity (e.g., $\mathrm{CN}^{-}$present in the measurement of $\mathrm{Ag}^{+}$), but a considerable gap will occur between the activity and concentration of the ions even in very dilute solutions. Under these circumstances the determination of ionic 
concentration may be problematic.

d. Substances interacting with the membrane itself, blocking the surface or changing its chemical composition (i.e., organic solvents for the liquid or poly(vinyl chloride) (PVC) membrane electrodes) are grouped as interferences or electrode poisons.

8. Ionic-strength or/and pH Adjustment Solution. These are solutions of high ionic strength added to samples and calibration solutions before measurement in order to achieve identical ionic strength. Often a fixed $\mathrm{pH}$ solution or buffer controls the hydrogen ion activity as well as ionic strength. In addition, complexing agents and other components are often added to minimize the effects of certain interferences.

9. Ion-selective Electrode (ISE). This is an electrochemical sensor, based on thin films or selective membranes as recognition elements, and is an electrochemical half-cell equivalent to other half-cells of the zeroth (inert metal in a redox electrolyte), 1st, 2 nd and 3 rd kinds. These devices are distinct from systems that involve redox reactions (electrodes of zeroth, 1st, 2nd and 3rd kinds), although they often contain a 2nd kind electrode as the "inner" or "internal" reference electrode. The potential difference response has, as its principal component, the Gibbs energy change associated with permselective mass transfer (by ion-exchange, solvent extraction or some other mechanism) across a phase boundary. The ISE must be used in conjunction with a reference electrode (i.e. "outer" or "external" reference electrode) to form a complete electrochemical cell. The measured potential differences (ISE versus outer reference electrode potentials) are linearly dependent on the logarithm of the activity of a given ion in solution. Comment: The term "ion-specific electrode" is not recommended. The term "specific" implies that the electrode does not respond to additional ions. Since no electrode is truly specific for one ion, the term "ion-selective" is recommended as more appropriate. "Selective ion-sensitive electrode" is a little-used term to describe an ion-selective electrode. "Principal" or "primary" ions are those which an electrode is designed to measure. It is never certain that the "principal" ion is most sensitively measured, e.g., nitrate ISEs.

10. Ion-selective Electrode Cell. An ion-selective electrode in conjunction with a reference electrode is an ISE cell. Generally, the cell contains two reference electrodes, internal and external, and the thin film or membrane recognition-transduction element. However, besides this conventional type of cell (with solution contact on both sides of the membrane) there are cell arrangements with wire contact to one side of the membrane (all solid state and coated wire types). Conventional writing $(2,6)$ of the cell is:

$$
\text { outer ref. || test solution | membrane | internal ref. }
$$

or

$$
\text { outer ref. || test solution | ion selective electrode }
$$

and the measured cell emf, $E$, (right electrode potential minus left electrode potential) has the form

$$
E=\text { constant } t+\frac{2.303 R T}{z_{A} F} \lg \left[a_{A}+K_{A, B}^{\text {pot }} a_{B}^{z_{A} / z_{B}}+K_{A, C}^{\text {pot }} a_{C}^{z_{A} / z_{c}}+\ldots\right]
$$

Terms in this equation are identified in Section III. Internal components of an ISE include solvent, $\mathrm{pH}$ buffer, salt of the sensed ion, and soluble salt of anion in equilibrium with the internal reference electrode of 2 nd kind. High-stability internal reference electrodes based on an inert metal electrode and a reversible redox couple are also common.

11. Isopotential Point. For an ion-selective electrode cell, there is often a particular activity of the measured ion for which the emf of the cell is independent of temperature. That activity, and the corresponding potential difference, defines the isopotential point. The specification of the ISE and outer reference electrode must be described. Comment: When an isothermal cell is used with identical reference electrodes, the isopotential point is the activity of sensed species that gives zero net membrane potential, e.g. sensed activity is the same in the inner and outer (test) solution. Calibration lines for different cell temperatures have different slopes, but intersect at a common activity point. Cells with temperature gradients are not recommended.

12. Membrane. This general term refers to a continuous layer, usually consisting of a semi-permeable material, with controlled permeability covering a structure, such as carbon or an inert metal, or separating two electrolyte solutions. This latter case is the most general form of an ISE. The membrane separates the internal components of the ISE from the test solution. The membrane of an 
ion selective electrode is responsible for the emf response and selectivity of the entire electrode (see chapter II for listing of membranes). Comment: Membranes of sensor electrodes are thought to be practically homogeneous, but an actual membrane may contain inhomogeneous regions, often at surfaces, and connected with materials and preparation methods used. Inhomogeneous regions include low dielectric polymer regions with few charge sites and regions with high local site densities. Surface regions of plasticized liquid membranes often are low in sites and high in plasticizer or exuded impurities.

13a. Membrane Sites. Membranes frequently contain built-in "fixed" charged sites (e.g., immobile $-\mathrm{SO}_{3}$ in polystyrenesulfonate), or intentionally added, hydrophobically trapped, "mobile" sites (e.g. tetraphenyl borate in plasticized poly(vinyl chloride)). Such membranes with charged sites are named sited membranes. Ions of opposite sign in the membrane are "counterions". Ions of the same sign as sites are not present in significant quantities, and are coions. Sited membranes are "permselective" to counterions i.e. only counterions exchange into the membrane and therefore have some mobility in the membrane bulk.

13b. Donnan Exclusion and Exclusion Failure. Sited membranes contain counterions whose aggregate charge exceeds that of sites. The excess counterion charge is balanced by a smaller (minority) number of "coions". In addition to the gross electroneutrality condition, the salt partition equilibrium expression

$$
\overline{a_{+} a_{-}}=K
$$

determines the exact concentration of minority coion species. The bar activities are salt ionic species in the membrane. Nonbar terms are the activities of ionic species of the salt in the bathing electrolyte solution. When site concentration is large, and bathing activities are small, almost all current is carried by counterions. This condition is Donnan exclusion since coions are nearly absent from the membrane. When $K$ is large, or bathing activities are large, or site concentration is small, then a membrane can contain large concentrations of coions, in the ultimate zero-site limit, equivalent in charge to counterion concentration. This condition is Donnan exclusion failure. There are two manifestations:

1. If the membrane is asymmetrically bathed, then salts diffuse through the membrane from high to low concentration sides.

2. If symmetrically bathed, but a voltage is applied across the membrane using the inner and outer reference electrodes through a high impedance circuit for example, then current is carried by both the counterions and coions.

14. Nernstian Response. Nernstian response occurs when an ion-selective electrode responds according to "local" thermodynamic equilibrium, over a given range of activity (or concentration). Nernstian response is revealed when a plot of the potential difference of the ISE cell (electrode, with an outer reference electrode) $v s$. the logarithm of the ionic activity of a given species $\left(a_{\mathrm{A}}\right)$ is linear with a slope of $2.303 \mathrm{RT} / \mathrm{z}_{\mathrm{A}} \mathrm{F}\left(59.16 / \mathrm{z}_{\mathrm{A}} \mathrm{mV}\right.$ per unit change of $\mathrm{p} a_{\mathrm{A}}$ at $\left.298.15 \mathrm{~K}\right)$. Nernstian response implies ideal sensitivity, but not necessarily ideal selectivity since interfering ions may also give Nernstian response when present as the sole potential determining species.

15. Potentiometric Selectivity Coefficient defines the ability of an ion-selective electrode to distinguish a particular ion from others. The selectivity coefficient, $\mathrm{K}_{\mathrm{A}, \mathrm{B}}^{\mathrm{pot}}$, is evaluated by means of the emf response of the ion-selective electrode in mixed solutions of the primary ion, $A$, and interfering ion, B (Fixed Interference Method) or less desirable, in separate solutions of A and B (Separate Solution Method). The activities of the primary ion, $A$, and the interfering ion, $B$, at which $K_{A, B}^{\text {pot }}$ is determined should always be specified, as the value of $\mathrm{K}_{\mathrm{A}, \mathrm{B}}^{\mathrm{pot}}$ is defined by the modified NikolskyEisenman equation, eq. (1). The smaller the value of $\mathrm{K}_{A, B}^{\mathrm{pot}}$ the greater the electrode's preference for the principal ion, $A$, as described below. Solution compositions and procedures for the determination are under consideration for separate IUPAC recommendations. Comment: The terms Selectivity Constant and Selectivity Factor are frequently used instead of selectivity coefficient. However, in order to standardize the terminology associated with ion-selective electrodes, use of the term selectivity coefficient is recommended, as is the fixed interference method for its evaluation 
(see section III D. 2). This selectivity coefficient is not identical to the similar term used in separation science.

16. Range and Span. The cell is said to have a range of response (in activity or concentration) between the lower and upper detection limits, determined from a plot of the cell potential difference $v s$. the logarithm of responsive ionic activity. The span is the corresponding emf difference (potential range in contrast to activity range) determined by projecting the lower (initial) and the upper (final) concentrations to the potential axis. Comment: The notion of span appears in the clinical literature, e.g. the normal span for $\mathrm{K}^{+}$in blood is a few millivolts which corresponds to the physiological activity range of the primary ion and interferences.

17a. Reference Electrode. An electrode which maintains a virtually constant potential with respect to the solution under the conditions prevailing in an electrochemical measurement, and which serves to permit the observation, measurement or control of the potential difference of the indicator (or test) or working electrode. Comment: In potentiometry, under zero current condition, the virtually constant potential difference is realized by assuring a constant composition solution in contact with the reference element, i.e. any electrode with small standard deviation can be used as a reference electrode (another ISE, electrodes of zero, 1st, 2nd, or 3rd kind). However, practical reference electrodes are generally nonpolarizable electrodes of the second kind, constructed so that their electrolyte solutions serve as salt bridges to the solutions under investigation. "Double" junction reference electrodes are recommended when the reference electrolyte contains ions that interfere with primary ion measurement or react with components of the test solution.

17b. Internal Reference Electrode. This is an "inner" reference electrode which is contained inside an ion-selective electrode assembly. Comment: The system frequently consists of a silver/silver chloride electrode in contact with an appropriate solution containing fixed concentrations of chloride and the ion for which the membrane is selective. This "inner" filling solution is in contact with the ionselective membrane.

18. Response Time. The time which elapses between the instant when an ion-selective electrode and a reference electrode (ISE cell) are brought into contact with a sample solution (or at which the activity of the ion of interest in a solution is changed) and the first instant at which the emf/time slope $(\Delta E / \Delta t)$ becomes equal to a limiting value selected on the basis of the experimental conditions and/or requirements concerning the accuracy (e.g. $0.6 \mathrm{mV} / \mathrm{min}$.). This concept is illustrated in Fig. 3. This is a convenient definition. In clinical applications (the physiological activity range corresponds to a small emf span), a smaller slope e.g. $0.1 \mathrm{mV} / \mathrm{min}$ may be chosen, provided the standard deviation of the response is less than the required slope. Comment: The previously defined response times $t_{95}$ (to $95 \%$ of the activity change corresponding span) and $t^{*}$ (to $1 \mathrm{mV}$ from the steady value) require prior knowledge of steady-state $E$ values that may not be available (7). These descriptive quantities underestimate practical response times of ion-selective electrodes in clinical applications where the total span may be less than $10 \mathrm{mV}$. To define the response time with $\Delta E / \Delta t$ (a rate of emf variation) seems to be the best choice among the non-ideal options. It can be related to $t_{95}$ and $t^{*}$ through mathematical models, provided the long-time potential determining processes have been identified.

19. Standard Addition or Known Addition Method. This is a procedure for the determination of the concentration of a particular species in a sample by adding known amounts of that species to the sample solution and recording the change in potential difference of an ion-selective electrode and suitable reference electrode cell. A variation of this method, viz. Analyte Addition, uses sequential addition of the unknown-concentration sample to a standard while recording cell emf changes. A method for data treatment (plotting the apparent concentration as derived from the cell emf versus the volume of standard or reagent added to the sample) and the standard addition is known as Gran Addition.

20 Standard Subtraction or Known Subtraction. This is a variation of the Standard Addition Method. In this procedure changes in the emf are employed, resulting from the addition of a known amount of a species which reacts stoichiometrically with the ion of interest (e.g., a complexing agent) to determine the original activity or concentration of the ion.

21. The "Suspension Effect" or Pallmann Effect (8-10) occurs when ISEs are used in concentrated, space-filled suspensions while the external reference electrode remains in the supernatant (suspension-free) solution. The suspensions are specifically solvent-swollen ion exchangers or other 
materials, like soils and clays, that concentrate ions by adsorption and absorption. Space-filled, gravity-packed suspensions act like a second phase and form apparently an interfacial potential difference (pd) with respect to the supernatant. The measured ion activity in the suspension differs from the value in the supernatant by the interfacial pd, and corresponds to a higher value approximating the activity inside the ion exchanger gel. The effect nearly disappears when the outer reference electrode is placed in the same region of the suspension as the sensor electrode. There are some changes in the junction potential differences of the reference electrode, between suspension and supernatant.

\section{CLASSIFICATION OF ION-SELECTIVE ELECTRODES}

\section{A. Primary Ion-Selective Electrodes}

1. Crystalline Electrodes may be homogeneous or heterogeneous. They contain mobile ions of one sign and fixed sites of opposite sign.

a. Homogeneous membrane electrodes are ion-selective electrodes in which the membrane is a crystalline material prepared from either a single compound or a homogeneous mixture of compounds (i.e., $\mathrm{A} \dot{g}_{2} \mathrm{~S}, \mathrm{AgI} / \mathrm{Ag}_{2} \mathrm{~S}$ ).

b. Heterogeneous membrane electrodes are formed when an active substance, or mixture of active substances, is mixed with an inert matrix, such as silicone rubber or PVC, or placed on hydrophobized graphite or conducting epoxy, to form the heterogeneous sensing membrane.

2. Non-crystalline Electrodes. In these electrodes, a support matrix, containing an ion exchanger (either cationic or anionic), a plasticizer solvent, and possibly an uncharged, selectivity-enhancing species, forms the ion-selective membrane which is usually interposed between two aqueous solutions. The support used can be either macroporous (e.g., poly(propylene carbonate) filter, glass frit, etc.) or microporous (e.g. "thirsty" glass or inert polymeric material such as PVC) yielding with the ion-exchanger and the solvent a "solidified" homogeneous mixture. These electrodes exhibit a response due to the presence of the ion-exchange material (typically selected from section $2 \mathrm{~b}$ ) in the membrane. The solvent-polymeric-membrane is an example.

a. Rigid, self-supporting, matrix electrodes (e.g., synthetic cross-linked polymer or glass electrodes) are ion-selective electrodes in which the sensing membrane is a thin polymer with fixed sites or a thin piece of glass. The chemical composition of the polymer (e.g. polystyrenesulfonate, sulfonated poly(tetrafluoroethylene), amino-poly(vinyl chloride)) or the glass determines the selectivity of the membrane.

b. Electrodes with mobile charged sites:

1. Positively charged, hydrophobic cations (e.g., those of quaternary ammonium salts or salts of substitutionally inert transition metal complexes such as the derivatives of 1,10phenanthroline) which, when dissolved in a suitable organic solvent and held in an inert support (e.g., poly(propylene carbonate) filter or PVC), provide membranes which are sensitive to changes in the activities of anions.

2. Negatively charged hydrophobic anions (e.g., of type $(\mathrm{RO})_{2} \mathrm{PO}_{2}-$, tetra-pchlorophenylborate, dinonylnaphthalene sulfonate) which, when dissolved in a suitable organic solvent and held in an inert support (e.g., poly(propylene carbonate) filter or PVC), provide membranes which are sensitive to changes in the activities of cations.

3. Uncharged "carrier" electrodes based on solutions of molecular complexing agents of cations (e.g., ion-dipole formers: antibiotics, macrocyclic compounds or other sequestering agents) and anions (e.g., adduct formers: organotin compounds, activated carbonyl compounds, and some porphyrins) which can be used in ion exchanger membrane preparations to give sensitivity and selectivity to certain cations and anions.

4. Hydrophobic ion pair electrodes of plasticized polymers (e.g., PVC) containing a dissolved hydrophobic ion pair (e.g., a cationic drug as cation tetraphenylborate, or anionic drug as tetraalkylammonium salt of an anion) respond to component ion activities in bathing electrolytes. Responses can be Nernstian to bathing electrolytes of the cation drug chloride, or sodium tetraphenylborate.

\section{B. Campound or Multiple Membrane (Multiple Laver) Ion-selective Electrodes}

1. Gas sensing electrodes are sensors composed of an indicator and a reference electrode in contact with a thin film of solution which is separated from the bulk of the sample solution by a gas- 
permeable membrane or an air gap. This intermediate solution interacts with the gaseous species (penetrated through the membrane or an air gap) in such a way as to produce a change in a measured constituent (e.g. the $\mathrm{H}^{+}$activity) of the intermediate solution. This change is then sensed by the ion-selective electrode and is related to the partial pressure of the gaseous species in the sample. (Note: In electrochemical literature the term gas electrode is used for the classical, redox-equilibrium-based gas electrodes as well, such as the hydrogen or the chlorine gas electrodes $\left(\mathrm{Pt}(s)\left|\mathrm{H}_{2}(g)\right| \mathrm{H}^{+}(a q)\right.$ or $\left.\mathrm{Pt}(s)\left|\mathrm{Cl}_{2}(g)\right| \mathrm{Cl}^{-}(a q)\right)$. These electrodes respond both to the partial pressure of the gas $\left(\mathrm{H}_{2}\right.$ or $\left.\mathrm{Cl}_{2}\right)$ and to the ionic activities $\left(\mathrm{H}^{+}\right.$or $\left.\mathrm{Cl}-\right)$. The Clark oxygen electrode fits under this classification although, in contrast to other gas sensors, it is an amperometric and not a potentiometric device.

2. Enzyme substrate electrodes are sensors in which an ion-selective electrode is covered with a coating containing an enzyme which causes the reaction of an organic or inorganic substance (substrate) to produce a species to which the electrode responds. Alternatively, the sensor could be covered with a layer of substrate which reacts with the enzyme, co-factor, or inhibitor to be assayed.

\section{Metal Contact or All-Solid-State Ion-selective Electrodes}

These electrodes do not have an inner electrolyte solution, and they depend on both ionic and electronic conductivities (mixed conductors). The inner reference electrode is replaced with an electronic conductor, e.g., a bromide sensor film $\mathrm{AgBr}$ may be reversibly contacted by $\mathrm{Ag}$, or anion sensors based on cation radical salts may be contacted by $\mathrm{Pt}(11,12)$. This configuration contrasts with normal membrane usage in which electrolyte solutions (inner filling solution and outer "test" solution) contact membranes.

\section{CONSTANTS AND SYMBOLS}

A. Equations For emf Responses of Ion-selective Electrode Cells and Definitions of $K_{A, B}^{\text {pot }}$ The Nikolsky-Eisenman equation

$$
E=\text { constant } t+\frac{2.303 R T}{z_{A} F} \lg \left[a_{A}+K_{A, B}^{p o t} a_{B}^{z_{A} / z_{B}}+K_{A, C}^{p o t} a_{C}^{z_{A} / z_{c}}+\ldots\right]
$$

$E \quad$ is the experimentally observed emf of a cell (in V) when the only variables are activities in the test solution;

$R \quad$ is the gas constant and is equal to $8.314510 \mathrm{~J} \mathrm{~K}^{-1} \mathrm{~mol}^{-1}$;

$T \quad$ is the absolute temperature (in $\mathrm{K}$ );

$F \quad$ is the Faraday constant and is equal to $9.6485309 \times 10^{4} \mathrm{C} \mathrm{mol}^{-1}$;

$a_{\mathrm{A}} \quad$ is the activity of the ion, $\mathrm{A}$;

$a_{\mathrm{B}} \& a_{\mathrm{C}} \quad$ are the activities of the interfering ions, $\mathrm{B}$ and $\mathrm{C}$, respectively;

$K_{A, B}^{p o t} \quad$ is the potentiometric selectivity coefficient for ion B with respect to the principal ion A

$z_{\mathrm{A}} \quad$ is the charge number: an integer with sign and magnitude corresponding to the charge of the principal ion, $\mathrm{A}$;

$z_{\mathrm{B}} \& z_{\mathrm{C}} \quad$ are charge numbers corresponding to the charge of interfering ions, $\mathrm{B}$ and $\mathrm{C}$, respectively. Sign of these charge numbers is the same as that of the principal ion.

\section{B. Ionic Strength of a Solution is Defined by:}

$$
I=\frac{1}{2} \sum_{i} c_{i} z_{i}^{2}
$$

$I \quad$ is the ionic strength;

$c_{\mathrm{i}} \quad$ is the anount concentration of the ion $\mathrm{i}$ (usually in moles per liter);

$z_{\mathrm{i}} \quad$ is the charge of the ion, $\mathrm{i}$.

\section{Other Symbols}

Sign conventions should be in accord with IUPAC recommendations $(2,6)$. 


\section{Methods For Determining $K_{A, B}^{\text {pot }}$}

1. Fixed Interference Method (FIM). The emf of a cell comprising an ion-selective electrode and a reference electrode (ISE cell) is measured with solutions of constant activity of interfering ion, $a_{\mathrm{B}}$, and varying activity of the primary ion. The emf values obtained are plotted $v s$. the logarithm of the activity of the primary ion $a_{\mathrm{A}}$. The intersection of the extrapolation of the linear portions of this plot indicates the value of $a_{A}$ which is to be used to calculate $K_{A, B}^{\text {pot }}$ from the Nikolsky-Eisenman equation

$$
\mathrm{K}_{\mathrm{A}, \mathrm{B}}^{\mathrm{pot}}=\frac{\mathrm{a}_{\mathrm{A}}}{\mathrm{a}_{\mathrm{B}}^{\mathrm{z}_{\mathrm{A}} / \mathrm{z}_{\mathrm{B}}}}
$$

2. Separate Solution Method (SSM). The emf of a cell comprising an ion-selective electrode and a reference electrode (ISE cell) is measured with each of two separate solutions, one containing the ion $\mathrm{A}$ of the activity $a_{\mathrm{A}}$ (but no $\mathrm{B}$ ), the other containing the ion $\mathrm{B}$ at the same activity $a_{\mathrm{B}}=a_{\mathrm{A}}$ (but no $\mathrm{A}$ ). If the measured values are $E_{\mathrm{A}}$ and $E_{\mathrm{B}}$, respectively, the value of may be calculated from the equation:

$$
\log K_{A, B}^{\text {pot }}=\frac{\left(E_{B}-E_{A}\right) z_{A} F}{2.303 R T}+\left(1-\frac{z_{A}}{z_{B}}\right) \lg a_{A}
$$

3. Separate Solution Method (SSM) II. The concentrations of a cell comprising an ionselective electrode and a reference electrode (ISE cell) are adjusted with each of two separate solutions, one containing the ion $\mathrm{A}$ of the activity $a_{\mathrm{A}}$ (but no $\mathrm{B}$ ), the other containing the ion $\mathrm{B}$ (but no $A$ ) of the activity as high as required to achieve the same measured cell voltage. From any pair of activities $a_{\mathrm{A}}$ and $a_{\mathrm{B}}$ giving the same cell voltage, the value of $\mathrm{K}_{\mathrm{A}, \mathrm{B}}^{\mathrm{pot}}$ may be calculated from the equation:

$$
\mathrm{K}_{\mathrm{A}, \mathrm{B}}^{\mathrm{pot}}=\frac{\mathrm{a}_{\mathrm{A}}}{\mathrm{a}_{\mathrm{A}}^{\mathrm{z}_{\mathrm{A}} / \mathrm{z}_{B}}}
$$

The FIM and SSM methods are recommended only when the electrode exhibits a Nernstian response to both principal and interfering ions. These methods are based on the assumption that plots of $E_{1}$ vs. $\lg \left(a_{\mathrm{A}} \mathrm{A}^{1 / \mid z_{\mathrm{A}}}\right)$ and $E_{2}$ vs. $\lg \left(a_{\mathrm{B}}{ }^{1 / / z_{\mathrm{B}}}\right)$ will be parallel and the vertical spacing is $(2.303 R T / F) \lg \mathrm{K}_{\mathrm{A}, \mathrm{B}}^{\text {pot }}$. However, the FIM can always be used to determine a minimum primary ion concentration level at which the effect at interference can be neglected. The actual conditions of the FIM method match the conditions under which the electrodes are used.

\section{REFERENCES}

1) "Recommendations-1975", Pure Appl. Chem., 48 (1976) 127-132.

2) "Quantities, Units and Symbols in Physical Chemistry", IUPAC "Green Book", Blackwell Scientific Publications. Oxford, UK 1988 and 1993.

3) P.C. Meier, D. Amman, W.E. Morf, W. Simon, in "Medical and Biological Applications of Electrochemical Devices", J. Koryta, Ed., Wiley, Chichester, 1980, p. 13.

4) P.C. Meier, Anal. Chim. Acta, 136 (1982) 363.

5) G.J. Moody, J.D.R. Thomas, Talanta, 19 (1972) 623.

6) "Compendium of Chemical Terminology IUPAC Recommendations", IUPAC "Gold Book", Blackwell Scientific Publications, Oxford, UK 1987.

7) E. Lindner, K. Tóth, E. Pungor, Pure Appl. Chem., 58 (1986) 469

8) H. Pallmann, Kolloidchem. Beih., 30 (1930) 334.

9) R. Bates, "Determination of pH, Theory and Practice", Wiley and Sons, N. Y. 1973.

10) R. P. Buck, Anal. Chem., 58 (1986) 1938.

11) R. P. Buck and P. Vanysek, "Interfacial Potentials at Mixed Conductor Interfaces: Nernst, NernstDonnan, Nernst Distribution and Generalizations", J. Electroanal. Chem. Interfacial Electrochem., 292 (1990) 73.

12) Junguo Zhao and R. P. Buck, "The Influence of Contact Materials on Electric Properties of Single Crystal AgBr", J. Electrochem. Soc., 136 (1989) 3342-3351. 